\title{
A Gel'fond type criterion in degree two
}

\author{
by \\ Benoit Arbour (Montréal) and Damien Roy (Ottawa)
}

1. Introduction. Let $\xi$ be any real number and let $n$ be a positive integer. Defining the height $H(P)$ of a polynomial $P$ as the largest absolute value of its coefficients, an application of the Dirichlet box principle shows that, for any real number $X \geq 1$, there exists a non-zero polynomial $P \in$ $\mathbb{Z}[T]$ of degree at most $n$ and height at most $X$ which satisfies

$$
|P(\xi)| \leq c X^{-n}
$$

for some suitable constant $c>0$ depending only on $\xi$ and $n$. Conversely, Gel'fond's criterion implies that there are constants $\tau=\tau(n)$ and $c=$ $c(\xi, n)>0$ with the property that if, for any real number $X \geq 1$, there exists a non-zero polynomial $P \in \mathbb{Z}[T]$ with

$$
\operatorname{deg}(P) \leq n, \quad H(P) \leq X, \quad|P(\xi)| \leq c X^{-\tau},
$$

then $\xi$ is algebraic over $\mathbb{Q}$ of degree at most $n$. For example, Brownawell's version of Gel'fond's criterion in [1] implies that the above statement holds with any $\tau>3 n$, and the more specific version proved by Davenport and Schmidt as Theorem $2 \mathrm{~b}$ of [4] shows that it holds with $\tau=2 n-1$. On the other hand, the above application of the Dirichlet box principle implies $\tau \geq n$. So, if we denote by $\tau_{n}$ the infimum of all admissible values of $\tau$ for a fixed $n \geq 1$, then we have $\tau_{1}=1$ and, in general,

$$
n \leq \tau_{n} \leq 2 n-1 \text {. }
$$

In the case of degree $n=2$, the study of a specific class of transcendental real numbers in [6] provides the sharper lower bound $\tau_{2} \geq \gamma^{2}$ where $\gamma=$ $(1+\sqrt{5}) / 2$ denotes the golden ratio (see Theorem 1.2 of [6]). Our main result below shows that we in fact have $\tau_{2}=\gamma^{2}$ by establishing the reverse inequality $\tau_{2} \leq \gamma^{2}$ :

Theorem. Let $\xi \in \mathbb{C}$. Assume that for any sufficiently large positive number $X$ there exists a non-zero polynomial $P \in \mathbb{Z}[T]$ of degree at most 2

2000 Mathematics Subject Classification: Primary 11J13; Secondary 11J82.

Work partially supported by NSERC and CICMA. 
and height at most $X$ such that

$$
|P(\xi)| \leq \frac{1}{4} X^{-\gamma^{2}}
$$

Then $\xi$ is algebraic over $\mathbb{Q}$ of degree at most 2 .

Comparing this statement with Theorem 1.2 of [6], we see that it is optimal up to the value of the multiplicative constant $1 / 4$ in (1). Although we do not know the best possible value for this constant, our argument will show that it can be replaced by any real number $c$ with $0<c<c_{0}=$ $\left(6 \cdot 2^{1 / \gamma}\right)^{-1 / \gamma} \cong 0.253$. As the reader will note, our proof, given in Section 3 below, has the same general structure as the proof of the main result of [3] and the proof of Theorem 1a of [4].

Following the method of Davenport and Schmidt in [4] combined with ideas from [2] and [7], we deduce the following result on simultaneous approximation of a real number by conjugate algebraic numbers:

Corollary. Let $\xi$ be a real number which is not algebraic over $\mathbb{Q}$ of degree at most 2 . Then there are arbitrarily large real numbers $Y \geq 1$ for which there exist an irreducible monic polynomial $P \in \mathbb{Z}[T]$ of degree 3 and an irreducible polynomial $Q \in \mathbb{Z}[T]$ of degree 2 , both of which have height at most $Y$ and admit at least two distinct real roots whose distance to $\xi$ is at most $c Y^{-(3-\gamma) / 2}$, with a constant $c$ depending only on $\xi$.

The proof of this corollary is postponed to Section 4 .

2. Preliminaries. We collect here several lemmas which we will need in the proof of the Theorem. The first one is a special case of the well known Gel'fond's lemma for which we computed the optimal values of the constants.

Lemma 1. Let $L, M \in \mathbb{C}[T]$ be polynomials of degree at most 1 . Then

$$
\frac{1}{\gamma} H(L) H(M) \leq H(L M) \leq 2 H(L) H(M) .
$$

The second result is an estimate for the resultant of two polynomials of small degree.

Lemma 2. Let $m, n \in\{1,2\}$, and let $P$ and $Q$ be non-zero polynomials in $\mathbb{Z}[T]$ with $\operatorname{deg}(P) \leq m$ and $\operatorname{deg}(Q) \leq n$. Then, for any complex number $\xi$,

$$
|\operatorname{Res}(P, Q)| \leq H(P)^{n} H(Q)^{m}\left(c(m, n) \frac{|P(\xi)|}{H(P)}+c(n, m) \frac{|Q(\xi)|}{H(Q)}\right)
$$

where $c(1,1)=1, c(1,2)=3, c(2,1)=1$ and $c(2,2)=6$.

The proof of the above statement is easily reduced to the case where $\operatorname{deg}(P)=m$ and $\operatorname{deg}(Q)=n$. The conclusion then follows by writing 
$\operatorname{Res}(P, Q)$ as a Sylvester determinant and by arguing as Brownawell in the proof of Lemma 1 of [1] to estimate this determinant.

The third lemma may be viewed, for example, as a special case of Lemma 13 of $[5]$.

Lemma 3. Let $P, Q \in \mathbb{Z}[T]$ be non-zero polynomials of degree at most 2 with greatest common divisor $L \in \mathbb{Z}[T]$ of degree 1 . Then, for any complex number $\xi$, we have

$$
H(L)|L(\xi)| \leq \gamma(H(P)|Q(\xi)|+H(Q)|P(\xi)|) .
$$

Proof. The quotients $P / L$ and $Q / L$ being relatively prime polynomials of $\mathbb{Z}[T]$, their resultant is a non-zero integer. Applying Lemma 2 with $m=$ $n=1$ and using Lemma 1 , we then deduce, if $L(\xi) \neq 0$,

$$
\begin{aligned}
1 \leq|\operatorname{Res}(P / L, Q / L)| & \leq H(P / L)|(Q / L)(\xi)|+H(Q / L)|(P / L)(\xi)| \\
& \leq \gamma \frac{H(P)}{H(L)} \frac{|Q(\xi)|}{|L(\xi)|}+\gamma \frac{H(Q)}{H(L)} \frac{|P(\xi)|}{|L(\xi)|} .
\end{aligned}
$$

Lemma 4. Let $\xi \in \mathbb{C}$ and let $P, Q, R \in \mathbb{C}[T]$ be arbitrary polynomials of degree at most 2 . Then, writing the coefficients of these polynomials as rows of a $3 \times 3$ matrix, we have

$$
|\operatorname{det}(P, Q, R)| \leq 2 H(P) H(Q) H(R)\left(\frac{|P(\xi)|}{H(P)}+\frac{|Q(\xi)|}{H(Q)}+\frac{|R(\xi)|}{H(R)}\right) .
$$

The above statement follows simply by observing, as in the proof of Lemma 4 of [3], that the determinant of the matrix does not change if, in this matrix, we replace the constant coefficients of $P, Q$ and $R$ by the values of these polynomials at $\xi$.

We also construct a sequence of "minimal polynomials" similarly to $\S 3$ of $[3]$ :

Lemma 5. Let $\xi \in \mathbb{C}$ with $[\mathbb{Q}(\xi): \mathbb{Q}]>2$. Then there exists a strictly increasing sequence $\left(X_{i}\right)_{i \geq 1}$ of positive integers and a sequence $\left(P_{i}\right)_{i \geq 1}$ of non-zero polynomials in $\mathbb{Z}[T]$ of degree at most 2 such that, for each $i \geq 1$ :

- $H\left(P_{i}\right)=X_{i}$

- $\left|P_{i+1}(\xi)\right|<\left|P_{i}(\xi)\right|$, $<X_{i+1}$

- $\left|P_{i}(\xi)\right| \leq|P(\xi)|$ for all $P \in \mathbb{Z}[T]$ with $\operatorname{deg}(P) \leq 2$ and $0<H(P)$

- $P_{i}$ and $P_{i+1}$ are linearly independent over $\mathbb{Q}$.

Proof. For each positive integer $X$, define $p_{X}$ to be the smallest value of $|P(\xi)|$ where $P \in \mathbb{Z}[T]$ is a non-zero polynomial of degree $\leq 2$ and height $\leq X$. This defines a non-decreasing sequence $p_{1} \geq p_{2} \geq \ldots$ of positive real numbers converging to 0 . Consider the sequence $X_{1}<X_{2}<\ldots$ of indices $X \geq 2$ for which $p_{X-1}>p_{X}$. For each $i \geq 1$, there exists a polynomial $P_{i} \in$ 
$\mathbb{Z}[T]$ of degree $\leq 2$ and height $X_{i}$ with $\left|P_{i}(\xi)\right|=p_{X_{i}}$. The sequences $\left(X_{i}\right)_{i \geq 1}$ and $\left(P_{i}\right)_{i \geq 1}$ clearly satisfy the first three conditions. The last condition follows from the fact that the polynomials $P_{i}$ are primitive of distinct height.

Lemma 6. Assume, in the notation of Lemma 5, that

$$
\lim _{i \rightarrow \infty} X_{i+1}\left|P_{i}(\xi)\right|=0 .
$$

Then there exist infinitely many indices $i \geq 2$ for which $P_{i-1}, P_{i}$ and $P_{i+1}$ are linearly independent over $\mathbb{Q}$.

Proof. Assume on the contrary that $P_{i-1}, P_{i}$ and $P_{i+1}$ are linearly dependent over $\mathbb{Q}$ for all $i \geq i_{0}$. Then the subspace $V$ of $\mathbb{Q}[T]$ generated by $P_{i-1}$ and $P_{i}$ is independent of $i$ for $i \geq i_{0}$. Let $\{P, Q\}$ be a basis of $V \cap \mathbb{Z}^{3}$. Then, for each $i \geq i_{0}$, we can write

$$
P_{i}=a_{i} P+b_{i} Q
$$

for some integers $a_{i}$ and $b_{i}$ of absolute value at most $c X_{i}$, with a constant $c>0$ depending only on $P$ and $Q$. Since $P_{i}$ and $P_{i+1}$ are linearly independent, we get

$$
1 \leq\left\|\begin{array}{cc}
a_{i} & b_{i} \\
a_{i+1} & b_{i+1}
\end{array}\right\|=\frac{\left|a_{i} P_{i+1}(\xi)-a_{i+1} P_{i}(\xi)\right|}{|Q(\xi)|} \leq \frac{2 c}{|Q(\xi)|} X_{i+1}\left|P_{i}(\xi)\right|
$$

in contradiction with the hypothesis as we let $i$ tend to infinity.

3. Proof of the Theorem. Let $c$ be a positive number and let $\xi$ be a complex number with $[\mathbb{Q}(\xi): \mathbb{Q}]>2$. Assume that, for any sufficiently large real number $X$, there exist a non-zero polynomial $P \in \mathbb{Z}[T]$ of degree $\leq 2$ and height $\leq X$ with $|P(\xi)| \leq c X^{-\gamma^{2}}$. We will show that these conditions imply $c \geq c_{0}=\left(6 \cdot 2^{1 / \gamma}\right)^{-1 / \gamma}>1 / 4$, thereby proving the Theorem.

Let $c_{1}$ be an arbitrary real number with $c_{1}>c$. By our hypotheses, the sequences $\left(X_{i}\right)_{i \geq 1}$ and $\left(P_{i}\right)_{i \geq 1}$ given by Lemma 5 satisfy

$$
\left|P_{i}(\xi)\right| \leq c X_{i+1}^{-\gamma^{2}}
$$

for any sufficiently large $i$. Then, by Lemma 6 , there exist infinitely many $i$ such that $P_{i-1}, P_{i}$ and $P_{i+1}$ are linearly independent. For such an index $i$, the determinant of these three polynomials is a non-zero integer and, applying Lemma 4, we deduce

$$
\begin{aligned}
1 \leq\left|\operatorname{det}\left(P_{i-1}, P_{i}, P_{i+1}\right)\right| & \leq 2 X_{i-1} X_{i} X_{i+1}\left(\frac{\left|P_{i-1}(\xi)\right|}{X_{i-1}}+\frac{\left|P_{i}(\xi)\right|}{X_{i}}+\frac{\left|P_{i+1}(\xi)\right|}{X_{i+1}}\right) \\
& \leq 2 c X_{i}^{-\gamma} X_{i+1}+4 c X_{i+1}^{1-\gamma} .
\end{aligned}
$$

Assuming that $i$ is sufficiently large, this implies

$$
X_{i}^{\gamma} \leq 2 c_{1} X_{i+1} \text {. }
$$


Suppose first that $P_{i}$ and $P_{i+1}$ are not relatively prime. Then their greatest common divisor is an irreducible polynomial $L \in \mathbb{Z}[T]$ of degree 1 , and Lemma 3 gives

$$
H(L)|L(\xi)| \leq \gamma\left(X_{i}\left|P_{i+1}(\xi)\right|+X_{i+1}\left|P_{i}(\xi)\right|\right) \leq 2 \gamma c X_{i+1}^{-\gamma} .
$$

Since $P_{i-1}, P_{i}$ and $P_{i+1}$ are linearly independent, the polynomial $L$ does not divide $P_{i-1}$ and so the resultant of $P_{i-1}$ and $L$ is a non-zero integer. Applying Lemma 2 then gives

$$
\begin{aligned}
1 \leq\left|\operatorname{Res}\left(P_{i-1}, L\right)\right| & \leq H\left(P_{i-1}\right) H(L)^{2}\left(\frac{\left|P_{i-1}(\xi)\right|}{H\left(P_{i-1}\right)}+3 \frac{|L(\xi)|}{H(L)}\right) \\
& \leq c X_{i}^{-\gamma^{2}} H(L)^{2}+3 X_{i-1} H(L)|L(\xi)| .
\end{aligned}
$$

Combining this with (3) and with the estimate $H(L) \leq \gamma H\left(P_{i}\right) \leq \gamma X_{i}$ coming from Lemma 1, we conclude that, in this case, the index $i$ is bounded.

Thus, assuming that $i$ is sufficiently large, the polynomials $P_{i}$ and $P_{i+1}$ are relatively prime and therefore their resultant is a non-zero integer. Using Lemma 2 we then find

$$
1 \leq\left|\operatorname{Res}\left(P_{i}, P_{i+1}\right)\right| \leq 6 X_{i} X_{i+1}\left(c X_{i} X_{i+2}^{-\gamma^{2}}+c X_{i+1}^{-\gamma}\right) \leq 6 c_{1} X_{i} X_{i+1}^{1-\gamma}
$$

since from (2), we have $c X_{i} \leq\left(c_{1}-c\right) X_{i+1}$ for large $i$. By (2) again, this implies

$$
1 \leq 6 c_{1}\left(2 c_{1}\right)^{1 / \gamma}
$$

and thus $c_{1} \geq c_{0}=\left(6 \cdot 2^{1 / \gamma}\right)^{-1 / \gamma}$. The choice of $c_{1}>c$ being arbitrary, this shows that $c \geq c_{0}$ as announced.

4. Proof of the Corollary. Let $\xi$ be as in the statement of the Corollary and let $V$ denote the real vector space of polynomials of degree at most 2 in $\mathbb{R}[T]$. It follows from the Theorem that there exist arbitrarily large real numbers $X$ for which the convex body $\mathcal{C}(X)$ of $V$ defined by

$$
\mathcal{C}(X)=\left\{P \in V ;|P(\xi)| \leq(1 / 4) X^{-\gamma^{2}},\left|P^{\prime}(\xi)\right| \leq c_{1} X \text { and }\left|P^{\prime \prime}(\xi)\right| \leq c_{1} X\right\}
$$

with $c_{1}=(1+|\xi|)^{-2}$ contains no non-zero integral polynomial. By Proposition 3.5 of [7] (a version of Mahler's theorem on polar reciprocal bodies), this implies that there exists a constant $c_{2}>1$ such that, for the same values of $X$, the convex body

$$
\mathcal{C}^{*}(X)=\left\{P \in V ;|P(\xi)| \leq c_{2} X^{-1},\left|P^{\prime}(\xi)\right| \leq c_{2} X^{-1} \text { and }\left|P^{\prime \prime}(\xi)\right| \leq c_{2} X^{\gamma^{2}}\right\}
$$

contains a basis of the lattice of integral polynomials in $V$.

Fix such an $X$ with $X \geq 1$, and let $\left\{P_{1}, P_{2}, P_{3}\right\} \subset \mathcal{C}^{*}(X)$ be a basis of $V \cap \mathbb{Z}[T]$. We now argue as in the proof of Proposition 9.1 of [7]. We put

$$
B(T)=T^{2}-1, \quad r=X^{-\left(1+\gamma^{2}\right) / 2}, \quad s=20 c_{2} X^{-1},
$$


and observe that any polynomial $S \in V$ with $H(S-B)<1 / 3$ admits at least two real roots in the interval $[-2,2]$ as such a polynomial takes positive values at \pm 2 and a negative value at 0 . We also note that, since $P_{i} \in \mathcal{C}^{*}(X)$, we have

$$
H\left(P_{i}(r T+\xi)\right) \leq c_{2} X^{-1} \quad(i=1,2,3) .
$$

Since $\left\{P_{1}, P_{2}, P_{3}\right\}$ is a basis of $V$ over $\mathbb{R}$, we may write

$$
(T-\xi)^{3}+s B\left(\frac{T-\xi}{r}\right)=T^{3}+\sum_{i=1}^{3} \theta_{i} P_{i}(T), \quad s B\left(\frac{T-\xi}{r}\right)=\sum_{i=1}^{3} \eta_{i} P_{i}(T)
$$

for some real numbers $\theta_{1}, \theta_{2}, \theta_{3}$ and $\eta_{1}, \eta_{2}, \eta_{3}$. For $i=1,2,3$, choose integers $a_{i}$ and $b_{i}$ with $\left|a_{i}-\theta_{i}\right| \leq 2$ and $\left|b_{i}-\eta_{i}\right| \leq 2$ so that the polynomials

$$
P(T)=T^{3}+\sum_{i=1}^{3} a_{i} P_{i}(T) \text { and } Q(T)=\sum_{i=1}^{3} b_{i} P_{i}(T)
$$

are respectively congruent to $T^{3}+2$ and $T^{2}+2$ modulo 4 . Then, by Eisenstein's criterion, $P$ and $Q$ are irreducible polynomials of $\mathbb{Z}[T]$. Moreover, we find

$$
\begin{aligned}
H\left(s^{-1} P(r T+\xi)-B(T)\right) & =s^{-1} H\left((r T)^{3}+\sum_{i=1}^{3}\left(a_{i}-\theta_{i}\right) P_{i}(r T+\xi)\right) \\
& \leq s^{-1} \max \left\{r^{3}, 6 c_{2} X^{-1}\right\}<1 / 3 .
\end{aligned}
$$

Then $P(r T+\xi)$ has at least two distinct real roots in the interval $[-2,2]$ and so $P$ has at least two real roots whose distance to $\xi$ is at most $2 r$. A similar but simpler computation shows that the same is true of the polynomial $Q$. Finally, the above estimate implies $H(P(r T+\xi)) \leq 4 s / 3$ and so $H(P) \leq$ $c_{3} X^{\gamma^{2}}$ for some constant $c_{3}>0$, and the same for $Q$. These polynomials thus satisfy the conclusion of the Corollary with $Y=c_{3} X^{\gamma^{2}}$ and an appropriate choice of $c$.

\section{References}

[1] W. D. Brownawell, Sequences of diophantine approximations, J. Number Theory 6 (1974), 11-21.

[2] Y. Bugeaud et O. Teulié, Approximation d'un nombre réel par des nombres algébriques de degré donné, Acta Arith. 93 (2000), 77-86.

[3] H. Davenport and W. M. Schmidt, Approximation to real numbers by quadratic irrationals, ibid. 13 (1967), 169-176.

[4] -, -, Approximation to real numbers by algebraic integers, ibid. 15 (1969), 393-416.

[5] M. Laurent and D. Roy, Criteria of algebraic independence with multiplicities and interpolation determinants, Trans. Amer. Math. Soc. 351 (1999), 1845-1870. 
[6] D. Roy, Approximation to real numbers by cubic algebraic integers I, Proc. London Math. Soc., to appear; arXiv:math.NT/0210181.

[7] D. Roy and M. Waldschmidt, Diophantine approximation by conjugate algebraic integers, Compositio Math., to appear; arXiv:math.NT/0207102.

Department of Mathematics

McGill University

805 Sherbrooke Ouest

Montréal, Québec H3A 2K6, Canada

E-mail: arbour@math.mcgill.ca
Département de Mathématiques Université d'Ottawa 585 King Edward Ottawa, Ontario K1N 6N5, Canada E-mail: droy@uottawa.ca 